\title{
Preface
}

This volume of proceedings gathers papers from the 1st Workshop on $\mathrm{Hu}-$ manities in the Semantic Web (WHiSe 2016), which was held on May 29, 2016 during the 13th ESWC Conference in Anissaras, Crete, Greece.

The decision to hold this workshop was a result of reflecting on the extent to which the Semantic Web community - through its vision, its technological offer and the large volumes of data it generates - has been serving the needs of historians, philologists, cultural critics, musicologists and other humanists. Prior efforts undertaken by the Semantic Web community to establish a communication channel with scholars in the Humanities had concentrated primarily on digital libraries and thesauri. While this has promoted the emergence of large semantically-structured digital collections and conceptual models such as CIDOC-CRM, EDM and FRBRoo, they left open questions as to how to aid the research of humanists who cannot rely on structured data generated en masse, unlike those whose use cases pass through social networks or large online media platforms. Is the race for Big Data mining cutting off these scholarly categories? Are there research challenges of interest that humanists will not pursue due to an opaque perception of what Linked Data is? What problems do Humanities users have in interacting with Semantic Web content and applications? And how can they help Semantic Web researchers support new modes of enquiry?

A response to such questions came from research groups primarily comprised of humanists with a focus on making their data structured and machine-readable. The fields of Classics, History and Archaeology have seen the emergence of a nascent Linked Pasts community that advocates an 'ecological' approach to few but widely-deployed ontologies to enhance discovery and visualisation. Similarly, the Musicology field is promoting Linked Data adoption through research networks such as Transforming Musicology. This prompted the organisers of this workshop to investigate avenues for promoting a dialogue between the two communities on equal terms, with each community serving both as investigators on research problems, and as providers of insights and solutions.

In this spirit, WHiSe was conceived so as to accommodate the modus operandi of each community on several fronts. The workshop included presentations of peer-reviewed papers - in the Computer Science style, as it were - and round-table discussions on central themes and novel research problems - in the Humanities style. Likewise, the program committee for peer reviews was equally balanced with acknowledged scholars from both communities. The topics of the papers presented, and included in this proceedings volume, spanned from use cases localised in specific regions implementing digital libraries, gazetteers and the like, to emergent ecosystems and research networks dealing with the preservation of entire histories of data. Round-table discussions encompassed research problems relating to data capture, especially dealing with vague or fragmentary sources, as well as re-thinking the ecologies of Linked Data for the Humanities in terms of what can guarantee their evolution and what risks holding them back. 
This first edition of WHiSe received twelve submissions; ten of these are presented in this volume, equally distributed between full papers of mature and validated research and short papers of early research, positions and accounts of community activation.

The editors would like to thank all the authors for their insightful contributions to WHiSe. Thanks are also due to the members of the program committee, for ensuring a quality standard of the workshop program through their reviews, and to the organisers of the ESWC 2016 conference for the chance and their continued support with setting up the workshop.

\section{Organising committee}

Alessandro Adamou, Knowledge Media Institute, The Open University, UK Enrico Daga, Knowledge Media Institute, The Open University, UK

Leif Isaksen, Department of History, Lancaster University, UK

\section{Program committee}

Carlo Allocca, The Open University

Elton Barker, The Open University

Francesca Benatti, The Open University

Gabriel Bodard, King's College

Kai-Christian Bruhn, mainzed

Benjamin Fields, Goldsmiths University of London

Nick Gibbins, University of Southampton

Asunción Gómez-Pérez, Universidad Politécnica de Madrid

Elena González-Blanco, Universidad Nacional de Educación a Distancia

Jorge Gracia, Universidad Politécnica de Madrid

Ethan Gruber, American Numismatic Society

Eero Hyvönen, Aalto University

Rinke Hoekstra, Vrije Universiteit Amsterdam

Laura Hollink, Centrum Wiskunde \& Informatica

Lorna Hughes, University of Glasgow

Antoine Isaac, Vrije Universiteit Amsterdam

Angeliki Lymberopoulou, The Open University

Elena Montiel, Universidad Politécnica de Madrid

Paul Mulholland, The Open University

Dominic Oldman, British Museum

Kevin R. Page, University of Oxford

Silvio Peroni, Alma Mater Studiorum Università di Bologna

Mia Ridge, The British Library

Rainer Simon, Austrian Institute of Technology

Ilaria Tiddi, The Open University

Daniel Vila-Suero, Universidad Politécnica de Madrid 